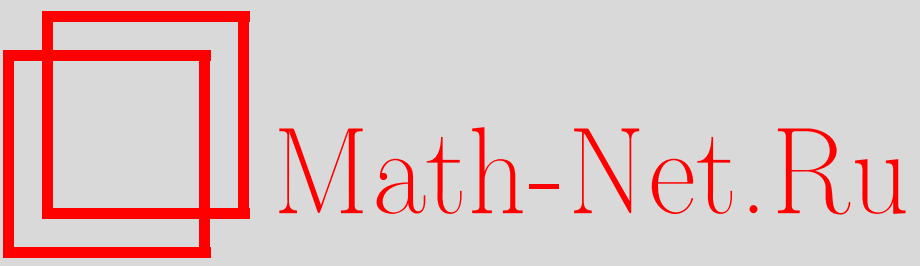

В. А. Мещеряков, Д. В. Мещеряков, Римановы поверхности некоторых статических дисперсионных моделей и проективные пространства, ТМФ, 2002, том 130, номер 3, 414-425

DOI: https://doi.org/10.4213/tmf309

Использование Общероссийского математического портала Math-Net.Ru подразумевает, что вы прочитали и согласны с пользовательским соглашением

http://www.mathnet.ru/rus/agreement

Параметры загрузки:

IP: 18.234 .197 .8

26 апреля 2023 г., 12:54:14

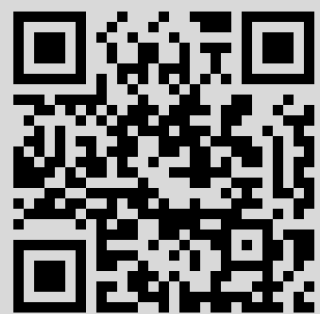


ТЕОРЕТИЧЕСКАЯ

И МАТЕМАТИЧЕСКАЯ

ФИЗИКА

Том 130, № 3

март, 2002

(C) 2002 г.

В. А. Мешеряков ${ }^{*}$, Д. В. Мещеряков ${ }^{\dagger}$

\section{РИМАНОВЫ ПОВЕРХНОСТИ НЕКОТОРЫХ СТАТИЧЕСКИХ ДИСПЕРСИОННЫХ МОДЕЛЕЙ И ПРОЕКТИВНЫЕ ПРОСТРАНСТВА}

Показано, что аналитическое продолжение элементов $S$-матрицы, являющихся мероморфными функциями энергии $\omega$ в комплексной плоскости с разрезами $(-\infty,-1]$, $[+1,+\infty)$, с физического листа на нефизические приводит к системе нелинейных разностных уравнений. Глобальный анализ этой системы выполнен в проективных пространствах $P_{N}$ и $P_{N+1}$. Обсуждается связь между пространствами $P_{N}$ и $P_{N+1}$. Получены некоторые частные решения исходной системы.

\section{1. ВВЕДЕНИЕ}

Задача о рассеянии адронов при низких энергиях до настояшего времени привлекает к себе внимание [1]. Успешное развитие КХД ставит вопрос о справедливости аналитических свойств амплитуд адрон-адронных процессов, доказанных ранее для сильных взаимодействий. Недавно в серии работ Омэ [2] было показано, что они остаются справедливыми и в КХД. Мы будем рассматривать нерелятивистский предел дисперсионных соотношений, известный под названием статические уравнения [3], и ограничимся изучением этого типа уравнений, сведя их к нелинейной краевой задаче [4]. Она имеет вид ряда условий на функции $S_{i}$ - матричные элементы $S$-матрицы.

УСловия 1.

A. $S_{i}(z)$ - мероморфные функции в комплексной плоскости $z$ с разрезами $(-\infty,-1]$, $[+1,+\infty)$, т.е. единственными особенностями этих функций в данной области являются нули и полюсы.

Б. $S_{i}^{*}(z)=S_{i}\left(z^{*}\right)$.

B. $\left|S_{i}(\omega+i 0)\right|^{2}=1$ при $\omega \geqslant 1, S_{i}(\omega+i 0)=\lim _{\epsilon \rightarrow+0} S_{i}(\omega+i \epsilon)$.

Г. $S_{i}(-z)=\sum_{j=1}^{N} A_{i j} S_{j}(z)$.

\footnotetext{
* Лаборатория теоретической физики им. Н. Н. Боголюбова, Объединенный институт ядерных исследований, Дубна, Московская обл., Россия

${ }^{\dagger}$ Московский государственный университет, Москва, Россия. E-mail: meshcher@recrep.phys.msu.su
} 
Действительные значения переменной $z$ являются полной энергией $\omega$ релятивистской частицы, рассеивающейся на фиксированном центре. Требование мероморфности функций $S_{i}(z)$ возникает как следствие статического предела задачи рассеяния [5]. Условие упругой унитарности $1 \mathrm{~B}$ справедливо лишь на правом разрезе плоскости $z$. На левом разрезе функции $S_{i}(z)$ заданы условиями перекрестной симметрии $1 \Gamma$. Матрица перекрестной симметрии $A$ определяется группой, относительно которой инвариантна $S$-матрица; для ряда групп она известна [4]. Цель настояшей работы состоит в формулировке способа изучения римановых поверхностей ряда статических дисперсионных моделей.

\section{2. АНАЛИТИЧЕСКОЕ ПРОДОЛЖЕНИЕ $S$-МАТРИЦЫ НА НЕФИЗИЧЕСКИЕ ЛИСТЫ}

Запишем условия 1 в матричной форме. Для этого введем столбец

$$
S^{(0)}(z)=\left[S_{1}(z), S_{2}(z), \ldots, S_{N}(z)\right]^{\mathrm{T}}
$$

где верхний индекс обозначает физический лист римановой поверхности $S$-матришы. Условия 1 А, Б, Г относятся к физическому листу, а условие унитарности 1В может быть продолжено на комплексные значения $\omega$. При этом оно, так же как условие $1 \mathrm{~B}$, имеет покомпонентный вид

$$
S_{i}^{(0)}(z) S_{i}^{(1)}(z)=1
$$

и осуществляет аналитическое продолжение $S$-матрицы на первый нефизический лист поверхности Римана. Матричная форма записи условия унитарности 1В получается при введении нелинейной операции инверсии $I$ согласно формуле

$$
I S(z)=\left[\frac{1}{S_{1}(z)}, \frac{1}{S_{2}(z)}, \ldots, \frac{1}{S_{N}(z)}\right] .
$$

В результате условия 1 приобретают следуюший вид.

Условия 2.

А. $S^{(0)}(z)$ - столбец $N$ мероморфных функций в комплексной плоскости $z$ с разрезами $(-\infty,-1],[+1,+\infty)$, т.е. единственными особенностями этих функций в данной области являются нули и полюсы.

Б. $S^{(0)^{*}}(z)=S^{(0)}\left(z^{*}\right)$.

B. $S^{(1)}(z)=I S^{(0)}(z)$.

Г. $S^{(0)}(-z)=A S^{(0)}(z)$.

Аналитическое продолжение на нефизические листы определим следующим образом:

$$
S^{(p)}(z)=(I A)^{p} S^{(0)}\left(z(-1)^{p}\right) .
$$


С помощью определения (1) условия унитарности $2 \mathrm{~B}$ и перекрестной симметрии $2 \Gamma$ легко продолжаются на нефизические листы:

$$
I S^{(p)}(z)=S^{(1-p)}(z), \quad A S^{(p)}(z)=S^{(-p)}(-z),
$$

и имеет место формула

$$
(I A)^{q} S^{(p)}(z)=S^{(q+p)}\left(z(-1)^{q}\right) .
$$

Мотивировка определения (1) содержится в известном решении [5] задачи, определяемой условиями 1 , для двухрядной матрицы

$$
A=\frac{1}{3}\left(\begin{array}{cc}
-1 & 4 \\
2 & 1
\end{array}\right)
$$

Это решение для $S$-матришы $S(z)$ имеет вид

$$
S(z)=\left(\begin{array}{l}
W(W-2) /\left(W^{2}-1\right) \\
W(W+1) /\left(W^{2}-1\right)
\end{array}\right) D(z),
$$

где $W=w+i \sqrt{z^{2}-1} \beta(z), w=(1 / \pi) \arcsin z, \beta(z)=-\beta(-z)$ - мероморфная функция и $D(z)=D(-z)$ фуннкция Бляшке переменной $\zeta=\left(1+i \sqrt{z^{2}-1}\right) / z$. Функция Бляшке имеет вид

$$
D(\zeta[z])=\zeta^{\lambda} \prod_{n} \frac{\left|\zeta_{n}\right|}{\zeta_{n}} \frac{\zeta_{n}-\zeta}{1-\zeta_{n}^{*} \zeta},
$$

где $\lambda$ - порядок нуля, а множество нулей $\left\{\zeta_{n}\right\}$ симметрично относительно начала координат и осей $\operatorname{Im} \zeta=0, \operatorname{Re} \zeta=0$, причем $\left|\zeta_{n}\right|<1$. Кроме решения (4) условия 1 допускают тривиальное решение - столбец одинаковых функций Бляшке

$$
S(z)=\left(\begin{array}{l}
1 \\
1
\end{array}\right) D(z) .
$$

Таким образом, условия 2 не определяют вид римановой поверхности $S(z)$ однозначно. В решении (4) риманова поверхность $S(z)$ бесконечнолистна за счет функции $w$, и справедливы равенства

$$
S^{(0)}(z)=\left.S(W)\right|_{|w| \leqslant 1 / 2}, \quad S^{( \pm n)}\left(z(-1)^{( \pm n)}\right)=\left.S(W)\right|_{|w \pm n| \leqslant 1 / 2},
$$

которые позволяют получить следуюшую форму уравнений (3):

$$
(I A)^{n} S(W)=S(W+n), \quad(A I)^{n} S(W)=S(W-n) .
$$

Уравнения (5) - система нелинейных автономных разностных уравнений, которые естественно назвать динамической формой статических дисперсионных соотношений. Поэтому и равенствам (3) можно присвоить это же наименование. В отличие от уравнений (5), они образуют систему нелинейных разностных уравнений, в которых аргументом является номер листа римановой поверхности, а энергетическая переменная $z$ фигурирует как параметр. 


\section{3. ФОРМУЛИРОВКА ЗАДАЧИ В ПРОЕКТИВНЫХ ПРОСТРАНСТВАХ}

На примере двухрядного решения (4) видно, что решение задачи, определяемой условиями 1 , вообще говоря, задается $N+1$ целыми функциями, $N$ из которых удовлетворяют условию перекрестной симметрии $1 \Gamma$, а последняя симметрична по $z$ и обеспечивает выполнение условия унитарности 1В. Условия $1 \mathrm{~A}$, Б, Г однородны и могут рассматриваться в проективных пространствах $P_{N-1}$ и $P_{N}$. Определим операцию нелинейной инверсии так, чтобы она была корректной в этих пространствах [6], и обозначим ее через $I_{p}$, где

$$
I_{p}=\prod_{j=1, i \neq j}^{m} S_{j}, \quad m=N-1, N .
$$

Переформулируем задачу, определяемую условиями 1 , в этих пространствах. В пространстве $P_{N-1}$ матрица перекрестной симметрии имеет заданный условиями 1 вид, а в пространстве $P_{N}$ ее порядок увеличивается на единицу, т.е.

$$
A_{N-1}=A, \quad A_{N}=\left(\begin{array}{cc}
A & 0 \\
0 & 1
\end{array}\right)
$$

где $A_{N}$ - блочная матрица. В результате вместо условий 1 получаем набор следуюших требований на столбец из $m$ функций.

\section{УСловия 3.}

А. $S^{(0)}(z)$ - столбец $m$ мероморфных функций в комплексной плоскости $z$ с разрезами $(-\infty,-1],[+1,+\infty)$, т.е. единственными особенностями этих функций в данной области являются нули и полюсы.

Б. $S^{(0)^{*}}(z)=S^{(0)}\left(z^{*}\right)$.

B. $S^{(1)}(z)=I_{p} S^{(0)}(z)$.

Г. $S^{(0)}(-z)=A_{m} S^{(0)}(z)$.

Проиллюстрируем схему решения двухрядного случая (4) на языке проективных пространств $P_{1}, P_{2}$. Обозначим через $\left(x_{0}, x_{1}\right)=\left(S_{1}, S_{2}\right)$ координаты точки $x$ в пространстве $P_{1}$. Введем на проективной прямой $P_{1}$ аффинную координату $X=x_{0} / x_{1}$. Полагая в формуле (1) $z=0$, получим закон продолжения координаты $X^{(0)}$ с физического листа на первый нефизический лист:

$$
X^{(1)}=\frac{2 X^{(0)}+1}{-X^{(0)}+4}
$$

Дробно-линейное преобразование (6) можно возвести в $n$-ю степень и из условия перекрестной симметрии 3 Г найти

$$
X^{(0)}=-2, \quad X^{(n)}=\frac{n-2}{n+1} .
$$

3 Теоретическая и математическая физика, т. 130, № 3, 2002 г. 
Таким образом, одно из условий перекрестной симметрии $3 Г$ является лишним. Вывод справедлив и для матриц перекрестной симметрии размерности $3 \times 3$.

Решение двухрядной задачи на прямой $P_{1}$ позволяет определить лишь отношение функций $S_{1}$ и $S_{2}$. Сами функции можно найти из решения ее на проективной плоскости $P_{2}$. Проективные координаты точки $x=\left(x_{0}, x_{1}, x_{2}\right)$ на $P_{2}$ запишем в базисе, явно учитьваюшем перекрестную симметрию:

$$
x_{0}=s-2 a, \quad x_{1}=s+a, \quad x_{2}=c,
$$

где $a$ - антисимметричная, а $s, c$ - симметричные функции $z$.

Рассматривая оператор $\left(I_{p} A_{2}\right)^{n}$ в базисе $s, a, c$, легко убедиться в том, что между $s, a, c$ сушествует соотношение

$$
s^{2}-a^{2}-s c=0,
$$

инвариантное относительно операторов $I_{p}$ и $A_{2}$ по отдельности.

Другими словами, уравнение (9) на $P_{2}$ определяет инвариантную кривую $C$, любая точка которой не покидает ее под действием преобразований $I_{p}$ и $A_{2}$. В базисе $x_{0}, x_{1}$, $x_{2}$ уравнение кривой $C$ имеет вид

$$
x_{1}^{2}+2 x_{0} x_{1}-2 x_{1} x_{2}-x_{0} x_{2}=0 .
$$

С помощью уравнений (7) и (10) легко найти, что

$$
\frac{x_{1}}{x_{2}}=\frac{n}{n-1},
$$

и тем самым определить полностью функции $S_{1}, S_{2}$. В процессе решения не было использовано условие унитарности $1 \mathrm{~B}$, учет которого позволяет полностью воспроизвести формулу (4).

Обсудим вопрос о соотношении описаний двухрядной задачи, определяемой условиями 1 , в пространствах $P_{1}$ и $P_{2}$. На проективной плоскости $P_{2}$ решение представлено инвариантной кривой (10). Она неприводима и, как всякая алгебраическая кривая второго порядка, рашиональна. В аффинных координатах она имеет вид

$$
x=\frac{x_{0}}{x_{2}}, \quad y=\frac{x_{1}}{x_{2}}, \quad x^{2}+2 x y-2 x-y=0 .
$$

Если построить пучок прямых $\lambda_{0} g_{0}+\lambda_{1} g_{1}$ с базисной точкой $\left(x_{0}, y_{0}\right)$ на кривой $(10)$, то координаты второй точки пересечения прямых пучка с кривой (10) будут рациональными функциями $k=\lambda_{1} / \lambda_{0}$ :

$$
x=\frac{-\left(x_{0}+2 y_{0}\right)+2+k}{1+2 k}, \quad y=y_{0}+k\left(x-x_{0}\right) .
$$


С помощью специально подобранной параметризации

$$
k=\frac{\left(-x_{0}-2 y_{0}+1\right) n+x_{0}+2 y_{0}-2}{n+1},
$$

зависящей от базисной точки пучка, функции $x$ и $y$ приводятся к формулам $(7),(11)$. При коллинеациях в пространстве $P_{2}$ (линейных преобразованиях с ненулевым детерминантом) пучок прямых ведет себя как проективное пространство $P_{1}$. Таким образом, проективное пространство $P_{1}$ представлено любым пучком прямых с базисной точкой на инвариантной кривой (10) пространства $P_{2}$.

Исследование и построение инвариантных многообразий для задачи, определяемой условиями 1, с размерностью $N \geqslant 3$ проводилось в работах [4] с помощью рядов в окрестности точек покоя динамических систем (5). Проективные пространства позволяют по-новому подойти к этой задаче.

Рассмотрим задачу, определяемую условиями 1 , с трехрядной матрицей

$$
A=\left(\begin{array}{ccc}
1 / 3 & -1 & 5 / 3 \\
-1 / 3 & 1 / 2 & 5 / 6 \\
1 / 3 & 1 / 2 & 1 / 6
\end{array}\right)
$$

которая описывает рассеяние двух частиц с угловыми моментами, равными единице. В пространстве $P_{3}$ матрица $A_{3}$ имеет три собственных значения +1 и одно -1 . Координаты точки $x$ в $P_{3}$ могут быть выражены через три симметричные $\left(s_{1}, s_{2}, s_{3}\right)$ и одну антисимметричную $(a)$ функции z с помощью обычной коллинеации (автоморфизма проективного пространства $\left.P_{3}\right)$ :

$$
x_{i}=b_{i j} s_{j}+b_{i 4} a .
$$

Построим в $P_{3}$ плоскость, инвариантную относительно линейного преобразования координат $x$, порождаемого матрищей $A_{3}$. Она имеет вид

$$
c_{0} x_{0}+c_{1} x_{1}+\left(2 c_{0}+c_{1}\right) x_{2}+c_{2} x_{3}=0 .
$$

Легко видеть, что частным случаем плоскости (13) будет плоскость $x_{1}+x_{2}=0$, которая инвариантна относительно преобразования $I_{p}$. Эта плоскость является пространством $P_{2}$, на котором задача, определяемая условиями 1 с матрицей $(12)$, сводится к решаемой двухрядной задаче [7]. Плоскость $x_{1}+x_{2}=0$ не проходит через точку покоя $\bar{x}=(1,1,1,1)$ динамической системы, определяемой условиями $3,-$ неподвижную точку преобразования (3). Если потребовать, чтобы точка $\bar{x}$ лежала на плоскости (13), то получим уравнение

$$
c_{0} x_{0}+c_{1} x_{1}+\left(2 c_{0}+c_{1}\right) x_{2}-\left(3 c_{0}-2 c_{1}\right) x_{3}=0 .
$$

Плоскость (14) под действием преобразования $I_{p}$ перейдет в поверхность третьего порядка в $P_{3}$, которая не инвариантна относительно преобразования $A_{3}$ :

$$
c_{0} x_{1} x_{2} x_{3}+c_{1} x_{0} x_{2} x_{3}+\left(2 c_{0}-c_{1}\right) x_{0} x_{1} x_{3}-\left(3 c_{0}+2 x_{1}\right) x_{0} x_{1} x_{2}=0 .
$$


Пересечение плоскости (14) и поверхности (15) определит плоскую пространственную кривую $C$, которая, вообще говоря, не инвариантна относительно преобразования $A_{3}$. Действительно, исключая $x_{3}$ из уравнений $(14),(15)$, получим однородное уравнение третьей степени $G\left(x_{0}, x_{1}, x_{2}\right)=0$. Функция $G$ в пространстве $P_{2}$ в базисе $s_{1}, s_{2}, a$ при произвольных $c_{0}, c_{1}$ будет содержать нечетные степени антисимметричной функции $a$. Коэффициент при $a$ - квадратичная форма по $s_{1}, s_{2}, a$. Инвариантность плоской пространственной кривой $C$ относительно преобразования $A_{3}$ требует обрашения этой квадратичной формы в нуль. Как всякое уравнение второй степени, оно определяет рациональные функции $s_{1}, s_{2}, a$ некоторого параметра $t$. Подставляя их в четную по $a$ часть функции $G\left(x_{0}, x_{1}, x_{2}\right)$, получим уравнение третьей степени относительно $t$, которое имеет, вообше говоря, три решения. Только тождественное обрашение его в нуль, т.е. приводимость $G$, гарантирует наличие инвариантной кривой. Приведем уравнение, определяющее коэффициенты $c_{0}, c_{1}$ :

$$
R_{x_{0}}\left(G, G_{x_{1}}^{\prime}\right) \equiv 0
$$

где $R_{x_{0}}$ - результант $G$ и $G_{x_{1}}^{\prime}$ по $x_{0}$. Из уравнения $(16)$ находим $c_{0}=-1, c_{1}=3$ и функцию

$$
G\left(x_{0}, x_{1}, x_{2}\right)=\left(-3 x_{1}^{2}+x_{0} x_{1}+3 x_{0} x_{2}-x_{1} x_{2}\right)\left(-x_{0}+x_{2}\right)=0,
$$

определяющую приводимую кривую $C$. Первый сомножитель в уравнении (17) инвариантен относительно преобразований $I_{p}$ и $A_{2}$ и совместно с уравнением (14) определяет известное решение [8] с конечным числом полюсов по $w$. Оно представлено в $P_{3}$ как пересечение плоскости

$$
-x_{0}+3 x_{1}+x_{2}-3 x_{3}=0
$$

и поверхности

$$
-3 x_{1}^{2}+x_{0} x_{1}+3 x_{0} x_{2}-x_{1} x_{2}=0 .
$$

Уравнение (19) с помошью равенства (18) можно записать в форме $x_{1} x_{3}=x_{0} x_{2}$, с помошью которой легко проверяется инвариантность равенства (18) относительно преобразования $I_{p}$. Второй сомножитель в уравнении (17) под действием преобразования $A_{3}$ переходит в $\left(-x_{1}+x_{2}\right)$, и в результате имеем вырожденную квадратичную форму $\left(-x_{0}+x_{2}\right)\left(-x_{1}+x_{2}\right)=0$, инвариантную относительно преобразований $I_{p}$ и $A_{3}$. Она определяет два пучка прямых, инвариантных относительно преобразования $I_{p}$ и переходящих друг в друга под действием преобразования $A_{3}$ :

$$
\begin{array}{ll}
x_{0}=x_{2}, & \frac{x_{0}}{x_{1}}=\frac{n+1 / 6}{n-7 / 6} ; \\
x_{1}=x_{2}, & \frac{x_{0}}{x_{1}}=\frac{n-3 / 2}{n+1 / 2} .
\end{array}
$$




\section{4. ЗАКЛЮЧЕНИЕ}

Нелинейная краевая задача о построении $N$-мерной (условие $1 \mathrm{~A}$ ), упруго-унитарной (условие 1 В) и кроссинг-симметричной (условие $1 \Gamma) S$-матрицы сформулирована в проективных пространствах $P_{N-1}$ и $P_{N}$. В пространстве $P_{N-1}$ ее можно рассматривать как результат проецирования исходной задачи, определяемой условиями 1 , из аффинного пространства $A_{N}$ в проективное пространство $P_{N-1}$, при котором игнорируется условие унитарности $1 \mathrm{~B}$. Условие аналитического продолжения $S$-матрицы на нефизические листы представлено в виде нелинейной автономной системы разностных уравнений, т.е. в динамической форме. Оно также может рассматриваться как нелинейное преобразование в пространствах $A_{N}, P_{N-1}, P_{N}$. Неподвижные точки этого преобразования включают, в частности, точку, соответствуюшую $S$-матрице без взаимодействия. В окрестности этой точки $S$-матрица изучалась с помощью степенных рядов по $1 / w$, которые иногда удается просуммировать [4]. Использование техники проективных пространств дает возможность глобального исследования решений - построения инвариантных подпространств, содержаших искомые решения. Инвариантные подпространства определяются однородными функциями в проективных пространствах $P_{N-1}$ и $P_{N}$, но не в аффинном пространстве $A_{N}$. Это утверждение расходится с выводом работы [9], согласно которому и в аффинном пространстве $A_{N}$ инвариантные подпространства определяются однородными функциями. Предложенная выше геометрическая интерпретация краевой задачи, определяемой условиями 1 , в проективных пространствах $P_{N-1}$ и $P_{N}$ и примеры, рассмотренные в работах [5], [8], указывают на необходимость отказаться от требования однородности функций, определяющих инвариантные подпространства в $A_{N}$.

В приложениях 1 и 2 продемонстрированы конкретные применения рассмотренной процедуры решения нелинейной краевой задачи, определяемой условиями 1.

ПРИЛОЖЕНИЕ 1

Двухрядная матрица перекрестной симметрии в группе $S U(2)$ имеет вид

$$
A_{2}=\frac{1}{2 l+1}\left(\begin{array}{cc}
-1 & 2 l+2 \\
2 l & 1
\end{array}\right), \quad l \in \mathbb{N} .
$$

Рассмотренная в статье матрица есть ее частный случай при $l=1$. Приведем схему вычисления обшего случая. На проективной прямой $P_{1}$ аффинная координата $X=x_{0} / x_{1}$ продолжается на первый нефизический лист по правилу

$$
X^{(1)}=\frac{2 l X^{(0)}+1}{-X^{(0)}+(2 l+2)}
$$

и вместе с условием перекрестной симметрии приводит к следующему значению $X^{(n)}$ :

$$
X^{(n)}=\frac{n-(l+1)}{n+l}, \quad X^{(0)}=-\left(1+\frac{1}{l}\right) .
$$


Тем самым на любом нефизическом листе определено отношение $x_{0} / x_{1}$ при $z=0$, и для построения функций $S_{1}, S_{2}$ необходимо найти лишш $x_{1} / x_{2}$. Обозначим это отношение через $\varphi=x_{1} / x_{2}$. Оно определяется из системы функциональных уравнений

$$
\begin{gathered}
\varphi^{(n)} \varphi^{(1-n)}=1 \\
\frac{\varphi^{(n)}}{\varphi^{(-n)}}=\frac{n+l}{n-l},
\end{gathered}
$$

которые следуют из условий унитарности и перекрестной симметрии (2) на нефизических листах. Здесь использованы те из равенств (2), которые не применялись при получении формул (20). Уравнение (21) имеет очевидное решение в кольце мероморфных функций

$$
\varphi^{(n)}=\frac{G(n)}{G(1-n)},
$$

где $G(n)$ - любая целая функция. Решение (23) может быть представлено в другой форме: $\ln \varphi^{(n)}=g(n-1 / 2)$, где $g(n-1 / 2)$ - нечетная функция своего аргумента. Последний вид функции $\ln \varphi^{(n)}$ удобен для решения уравнения $(22)$, которое легко приводится к виду

$$
g(n+1)+g(n)=\ln \frac{n+1 / 2+l}{n+1 / 2-l} .
$$

Частное решение неоднородного разностного уравнения (24) может быть найдено с помошью последовательного применения замен неизвестной функции согласно формуле

$$
g_{m}(n)=g_{m+1}(n)+\ln \frac{n+(-1)^{m} \alpha_{m+1}}{n-(-1)^{m} \alpha_{m+1}}
$$

где $\alpha_{k}=1 / 2+l-k$ и $g_{0}(n)=g(n)$. Функция $g_{k}(n)$ удовлетворяет уравнению

$$
g_{k}(n+1)+g_{k}(n)=\ln \frac{n+1 / 2+(-1)^{k}(l-k)}{n+1 / 2-(-1)^{k}(l-k)}
$$

и очевидно, что $g_{l}(n+1)+g_{l}(n)=0$. Общее решение этого однородного уравнения определяет функцию $D(z)$ в формуле $(4)$, которая не влияет на вид инвариантных связей между $x_{0}, x_{1}, x_{2}$. Поэтому положим $g_{l}=0$ и получим для $\varphi^{(n)}$ выражение

$$
\varphi^{(n)}=\prod_{m=1}^{l} \frac{n-1 / 2-(-1)^{m}(1 / 2+l-m)}{n-1 / 2+(-1)^{m}(1 / 2+l-m)} .
$$

Исключая из уравнений (20) и (25) параметр $n$, получим уравнение, определяемое однородным полиномом степени $(l+1)$ от $x_{0}, x_{1}, x_{2}$. При $l=1$ оно дает уравнение $(10)$. 
ПРИЛОЖЕНИЕ 2

Применим разработанный метод к задаче рассеяния псевдоскалярного мезона с единичным угловым моментом на фиксированном нуклоне с таким же угловым моментом. В этом случае матрица кроссинг-симметрии дается выражением (12). Разложим столбец $S(z)$ по собственным векторам матрицы $A$ :

$$
S(z)=s_{1}(z)\left(\begin{array}{l}
1 \\
1 \\
1
\end{array}\right)+\frac{1}{4} s_{2}(z)\left(\begin{array}{c}
15 \\
-5 \\
3
\end{array}\right)+2 \psi(z)\left(\begin{array}{l}
-2 \\
-1 \\
-1
\end{array}\right) .
$$

Функциональное уравнение (3) при $q=1, p=0$ в пределе $z \rightarrow \infty$ определяет фиксированные точки (точки покоя) задачи. Возврашаясь от базиса $s_{1}(z), s_{2}(z), \psi(z)$ к столбцу $S(z)$, имеем

$$
S= \pm i\left(\begin{array}{c}
-(2 \pm \sqrt{5}) \\
-(1 \pm \sqrt{5}) / 2 \\
(1 \pm \sqrt{5}) / 2
\end{array}\right)
$$

Из (27) видно, что все точки покоя лежат в плоскости $S_{2}+S_{3}=0$. Данная плоскость является инвариантом преобразований инверсии $I$ и кроссинг-симметрии $A$. Трехрядная матрища кроссинг-симметрии $(12)$ на плоскости $S_{2}+S_{3}=0$ переходит в двухрядную матрицу

$$
A_{2}=\frac{1}{3}\left(\begin{array}{cc}
1 & -8 \\
-1 & -1
\end{array}\right)
$$

и задача сводится к отысканию двух функций $S_{1}(z), S_{2}(z)$. Полагая $z=0$ и вводя $X^{(k)}=$ $S_{1}^{(k)} / S_{2}^{(k)}$, где $k$ - номер листа римановой поверхности, получаем, что переход от физического листа $k=0$ к листу с номером $n$ есть дробно-линейное преобразование:

$$
X^{(n)}=\sqrt{5} \frac{\sqrt{5}\left(X^{(0)}-2\right)\left(y_{-}^{n}-y_{+}^{n}\right)+\left(X^{(0)}+4\right)\left(y_{-}^{n}+y_{+}^{n}\right)}{\left(X^{(0)}+4\right)\left(y_{-}^{n}-y_{+}^{n}\right)+\sqrt{5}\left(X^{(0)}-2\right)\left(y_{-}^{n}+y_{+}^{n}\right)}
$$

где $y_{ \pm}=(3 \pm \sqrt{5}) / 2$. Требования унитарности и кроссинг-симметрии $X^{(n)}$ приводят к следуюшему условию для определения $X^{(0)}$ :

$$
\left(X^{(0)}-2\right)\left(X^{(0)}+4\right)=0 .
$$

Следовательно, мы получаем два различных решения, совместимых с требованиями унитарности и кроссинг-симметрии: $X^{(0)}=2$ и $X^{(0)}=-4$.

Таким образом, на любом нефизическом листе римановой поверхности задачи, определяемой условиями 2 с матрицей (28), отношение $S_{1} / S_{2}$ определено при $z=0$, и для построения $S_{1}$ и $S_{2}$ достаточно найти любую из этих функций. Введем обозначение 
$S_{2}(n)=\Phi(n)=-s_{2}(n)+\psi(n)$, где функции $s_{2}$ и $\psi$ были введены в (26). Эта функция удовлетворяет системе функциональных уравнений

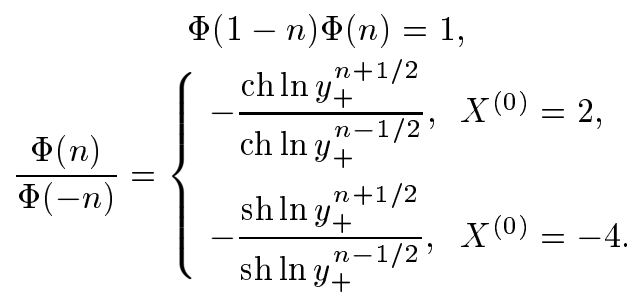

При получении уравнения (32) было использовано соотношение (29). Уравнение (31) имеет решение

$$
\Phi(n)=e^{g(n-1 / 2)},
$$

где $g(n)$ - любая нечетная функция, $g(n)=-g(-n)$. Подставляя (33) в (32), после замены $n \rightarrow n+1 / 2$ получаем разностное уравнение для определения неизвестной функции $g(n):$

$$
g(n+1)+g(n)= \begin{cases}\ln (-1) \frac{\operatorname{ch}\left((n+1) \ln y_{+}\right)}{\operatorname{ch}\left(n \ln y_{+}\right)}, & X^{(0)}=2, \\ \ln (-1) \frac{\operatorname{sh}\left((n+1) \ln y_{+}\right)}{\operatorname{sh}\left(n \ln y_{+}\right)}, & X^{(0)}=-4 .\end{cases}
$$

Решая уравнение (34) методом последовательных функциональных замен, получаем

$$
g(n)=g_{-1}(n)+g_{\infty}(n)+\sum_{m=0}^{\infty} G_{m}(n),
$$

где $g_{\infty}(n)=n \ln y_{+}$и

$$
G_{m}(n)=\left\{\begin{array}{l}
\ln \frac{\operatorname{ch}\left((n+1+2 m) \ln y_{+}\right) \operatorname{ch}\left((n-2(m+1)) \ln y_{+}\right)}{\operatorname{ch}\left((n-1-2 m) \ln y_{+}\right) \operatorname{ch}\left((n+2(m+1)) \ln y_{+}\right)}, \quad X^{(0)}=2, \\
\ln \frac{\operatorname{sh}\left((n+1+2 m) \ln y_{+}\right) \operatorname{sh}\left((n-2(m+1)) \ln y_{+}\right)}{\operatorname{sh}\left((n-1-2 m) \ln y_{+}\right) \operatorname{sh}\left((n+2(m+1)) \ln y_{+}\right)}, \quad X^{(0)}=-4 .
\end{array}\right.
$$

Слагаемое $g_{-1}(n)$ введено для учета множителя $(-1)$ в уравнении $(34)$. Введем обозначение $\xi(n)=e^{g_{-1}(n)}$. Тогда функция $\xi(n)$ есть решение системы функциональных уравнений

$$
\xi(n+1) \xi(n)=-1, \quad \xi(n) \xi(-n)=1 .
$$

Обшее решение этой системы выражается через тэта-функции. Здесь мы ограничимся вырожденным случаем

$$
\xi(n)=\operatorname{tg}\left[\frac{\pi}{2}\left(n+\frac{1}{2}\right)\right] .
$$


Используем теперь условие унитарности 1В. В результате функция $n$, как функция комплексной переменной $z$, является решением краевой задачи и имеет следующий вид:

$$
n(z)=\frac{1}{\pi} \arcsin z+i \sqrt{z^{2}-1} \beta(z)
$$

где $\beta(z)=-\beta(-z)-$ произвольная мероморфная функция. Из уравнения (39) следует, что риманова поверхность рассматриваемой модели имеет алгебраические точки ветвления при $z= \pm 1$ и логарифмическую точку ветвления на бесконечности. Теперь формулы (29), (30), (33)-(36), (38), (39) дают обшее решение задачи, определяемой условиями 1 , для матрицы кроссинг-симметрии (28).

\section{Список литературы}

[1] A. L. Machovariani, A. G. Rysetsky. Nucl. Phys. A. 1990. V. 515. P. 621.

[2] R. Oehme. Phys. Rev. D. 1990. V. 42. P. 4209; Phys. Lett. B. 1990. V. 252. P. 14; J. Mod. Phys. A. 1995. V. 10. P. 1995.

[3] G. F. Chew, F. E. Low. Phys. Rev. 1956. V. 101. P. 1570.

[4] В. А. Мещеряков. Статистические модели в дискретном подходе. Препринт № Р-2369. Дубна: ОИЯИ, 1965; В. И. Журавлев, В. А. Мещеряков. ЭЧАЯ. 1974. Т. 5. С. 173.

[5] G. Wanders. Nuovo Cimento. 1962. V. 23. Р. 816; B. А. Мещеряков. ЖЭТФ. 1966. Т. 51. C. 648 .

[6] V. A. Meshcheryakov. Solutions of nonlinear problems of dispersion relations in projective spaces. In: Proc. of Symp. in Ahrenshoop. Preprint PHE81-7. Zeuthen: Institut fur Hoohenenergiephysik Akademie der Wisserschaften der DDR, 1981. P. 44.

[7] В. И. Журавлев, В. А. Мещеряков, К. В. Рерих. ЯФ. 1969. Т. 10. С. 168.

[8] В. А. Мещеряков. ДАН СССР. 1967. Т. 174. С. 1054.

[9] M. Froissart, R. Omnes. Compt. Rend. Acad. Sci. 1957. V. 245. P. 2203.

Поступила в редакцию 10. VII.2001 г., после доработки 17.Х.2001 г. 